\title{
Time will tell: Deficits of temporal-information processing in patients with visual field loss
}

\author{
Dorothe A. Poggel $^{\mathrm{a}, \mathrm{b}, *}$, Bernhard Treutwein ${ }^{\mathrm{c}}$, Hans Strasburger ${ }^{\mathrm{a}, \mathrm{b}}$ \\ ${ }^{a}$ Generation Research Program (GRP), Human Science Center, Ludwig-Maximilian University Munich, Prof.-Max-Lange-Platz. 11, \\ 83646 Bad Tölz, Germany \\ ${ }^{b}$ Department of Medical Psychology and Medical Sociology, University of Göttingen, Waldweg 37, 37073 Göttingen, Germany \\ c IuK, Ludwig-Maximilian University Munich, Geschwister-Scholl-Platz 1, 80539 München, Germany
}

\section{A R T I C L E I N F O}

\section{Article history:}

Accepted 17 Oct. 2010

Available online: 23 Oct. 2010

Keywords:

temporal resolution,

visual field defect,

topography,

reaction time,

blindness,

perimetry

\begin{abstract}
A B S T R A C T
Visual field loss after brain lesions is commonly determined using perimetric tests of light detection (perimetry). Many patients with visual field defects complain about perceptual difficulties in areas that are perimetrically normal. To look at a potential cause for such difficulties, we topographically determined temporal characteristics of visual information processing in those patients and compared them to those of healthy subjects.

In nine patients with visual field loss we measured thresholds of double-pulse resolution (DPR), i.e. the minimum perceivable duration of a temporal gap between two light pulses, at eccentricities up to $20^{\circ}$. Furthermore, high-resolution maps of visual reaction times (RT) were obtained in a computer based campimetric test. Performance was compared to healthy controls from a cross-sectional study of temporal perception across the life span. (Toelz Temporal Topography Study).

Compared to healthy subjects, DPR thresholds and RTs in patients are elevated in the entire visual field, including areas that are perimetrically intact. Performance on temporal variables depends on the degree of intactness of the respective visual field position. DPR thresholds correlate considerably with RTs, and both parameters increase with eccentricity. However, whereas DPR thresholds are increased around blind regions relative to the intact field, this is not the case for RTs.

Temporal processing in patients with cerebral vision loss is impaired to a certain extent independently from perimetric light-detection performance. This may partly explain reported subjective perceptual problems. The findings may have important implications for visual rehabilitation, i.e. the potential generalization of light detection training to temporal processing performance.
\end{abstract}

\footnotetext{
Corresponding Author. Department of Medical Psychology and Medical Sociology, University of Göttingen, Waldweg 37, 37073 Göttingen; Germany. Fax: +49-551-3913558

E-mail address: dapoggel@gmail.com (D.A. Poggel).

Abbreviations: DPR: double-pulse resolution; RT: reaction time, CFF: Critical flicker fusion:
} 


\section{Introduction}

Conventional perimetry of light detection is the method of choice for evaluating the intactness of the visual field (Harrington, 1981). However, many patients with visual field defects complain about difficulties of visual perception that cannot be identified with standard perimetry or other common clinical measures of visual function, e.g. in glaucoma (Horn et al., 1999; Tyler, 1981) but also after cortical damage (Castelo-Branco et al., 2006; Poggel, 2002). Hence, without additional testing, a patients' complaints may appear groundless when in fact they may be a sign of undetected damage to the visual pathway or of beginning stages of a progressing disease.

Topographical deviations between different visual functions may explain why some forms of visual impairment remain undetected in standard clinical testing (Bachmann and Fahle, 2000; Castelo-Branco et al., 2006). The variability of perimetric maps of different psychophysical measures in healthy individuals is not surprising given the high degree of functional specialization within the visual pathway, even at the earliest stages (e.g. between magno- and parvo-cellular systems (Callaway, 2005; DeYoe and Van Essen, 1988) and ventral vs. dorsal pathways beyond the primary visual cortex (Goodale et al., 2005; Mishkin and Ungerleider, 1982)). Hence, different test parameters may preferably reflect the function of different pathways in the visual system, resulting in different topographical distributions in the visual field (Silva et al., 2008). For instance, perimetric maps of luminance detection thresholds show little relation to the respective topographies of reaction times or letter contrast sensitivity (see Gothe et al., 2000; Poggel et al., 2004a; Poggel and Strasburger, 2004; Strasburger and Rentschler, 1996; Poggel et al., submitted). The situation becomes even more complex in patients with damage to the visual system because it is possible that lesions affect only parts of the visual pathway while others remain intact, thus again resulting in different topographic features of visual field maps (Bachmann and Fahle, 2000; Castelo-Branco et al., 2006).

One dimension of visual perception that is mostly ignored in conventional clinical testing is temporal processing of visual information. An impairment of temporal-information processing may be caused by the same lesion that induces loss of other perceptual function, e.g. vision loss after damage to the primary visual cortex. Even without visual field defects, temporal processing may be impaired, however, resulting for instance in problems of temporal resolution or motion perception (Castelo-Branco et al., 2009b; Mendes et al., 2005). Such "secondary" defects would not normally be detected by conventional perimetry. The neural processing of time-related information and its relationship with sensory processes and their dynamic properties are still largely unknown or not well understood (Ivry and Spencer, 2004; Mauk and Buonomano, 2004; Poggel et al., 2004a; Poggel and Strasburger, 2004; Poggel et al., submitted).

Processing temporal information proper, like estimating the duration of some event, seems a universal, supra-modal, and highly flexible capacity of the brain that is most likely supported by distributed cortical and subcortical networks (see Wittmann, 1999; Wittmann, 2009, for a review). Networks of temporal-information processing are probably closely connected to perceptual functions, similar to the close interaction between perceptual and attentional functions. For example, some deficits of attention, e.g. spatial neglect, resemble perceptual deficits, and specific tests must be employed to differentiate attentional and perceptual impairments (Muller-Oehring et al., 2003).

Temporal processing of visual information is performed at several levels, spanning from presumably low-level properties like those assessed by flicker fusion, to higher cognitive functions like the subjective estimation of temporal durations (see Wittmann, 1999). Here we are interested in two low-level process characteristics: temporal resolution and reaction times. The purpose of the present study is to examine whether and how these functions are affected by visual system lesions and whether their topographic distributions deviate from that of light detection performance.

Some non-standard perimetric techniques are available for mapping temporal visual functions, including component perimetry (Bachmann and Fahle, 2000) and flicker perimetric methods (see McKendrick, 2005; Rota-Bartelink, 1999, for a review). Component perimetry simultaneously presents stimuli of a certain category across the visual field and tests the subjective perception of the defective area, particularly in patients with post-geniculate visual pathway lesions. While this method allows a fast test and an overview of potential defects, it does not include threshold testing or consider differences in temporal resolution across the visual field. To improve our understanding of the relationship between temporal processing and basic visual processes like light detection, it would be of interest to compare in more detail maps of conventional light detection thresholds with those of thresholds of temporal resolution. The various flicker perimetry approaches do allow for detailed mapping and also threshold testing. Their clinical application aims at uncovering retinal or other eye diseases, however, but they are not considered a standard part of ophthalmic examination in patients with post-geniculate defects. In addition, there are some methodological problems involved in the use of flicker stimulation (see below).

Temporal resolution of visual stimuli has typically been measured by the critical flicker frequency (or flicker fusion, $\mathrm{CFF}$ ), i.e. the threshold frequency at which a train of light pulses is perceived as non-flickering (Otto, 1987; Treutwein, 1989; Treutwein and Rentschler, 1992; Tyler, 1985; Tyler, 1987; Tyler and Hamer, 1990; Tyler and Hamer, 1993; Watson, 1986). Treutwein \& Rentschler (1992) suggested using double pulses to measure temporal resolution by a transient stimulus - rather than by the steady-state response as in the CFF (see Rashbass, 1970) thus avoiding adaptation to flicker (Tyler, 1985; Tyler, 1987; Tyler and Hamer, 1990; Tyler and Hamer, 1993). Treutwein developed an adaptive maximum-likelihood method for measuring double pulse resolution (DPR) thresholds at multiple locations within one test (Treutwein, 1989; Treutwein and Rentschler, 1992). There are nine white square patches presented on a dark screen (one in the center, the other eight on a circle around it (see Methods). The presentation of the target patch is interrupted by a short temporal gap, i.e. it is a double pulse whereas the distracters are presented continuously. Treutwein excluded the influence of confounding cues like brightness differences from temporal summation or asynchrony in the test (Treutwein and Rentschler, 1992) so that the subject's judgments reflect exclusively the minimal perceived temporal gap between light pulses at which the 
stimuli can be perceived as two events (i.e. as flickering). As such, DPR performance might particularly reflect magnocellular pathway function and beyond the primary visual cortex area MT involvement both of which seem specialized in processing time-related information.

Poggel et al. (Poggel et al., 2004a; Poggel and Strasburger, 2004; Poggel et al., 2006b) measured DPR thresholds across the visual field up to $20^{\circ}$ eccentricity in a large sample of healthy subjects across the lifespan. DPR thresholds increased (i.e. sensitivity decreased) systematically and significantly with eccentricity, with a particularly steep rise within the central $5^{\circ}$ radius. In part the increase of DPR thresholds stemmed from the fact that stimulus sizes were not scaled to compensate for the peripherally lower cortical magnification factor (Horton and Hoyt, 1991; see Drasdo, 1991 for a review $\}$. To another part, however, the increase was explained by effects of sustained spatial attention through an increased size of the attentional focus and thus more diffuse attention (Poggel et al., 2006b). When these two factors were taken into account in a quantitative model, the temporal sensitivity in healthy subjects as measured by DPR thresholds was almost homogeneous within the inner $5^{\circ}$ to $20^{\circ}$ radius. DPR thresholds vary, however, over the life span, and the periphery of the visual field is affected by the aging process more than the visual field center (Poggel et al., 2004a; Poggel et al., submitted).

Another important measure of low-level temporalinformation processing are simple reaction times (RTs) to light stimuli which have been examined in a wide range of studies on vision and visual attention (Becker et al., 2005; Luce, 1986; Schiefer et al., 2001; Teichner and Krebs, 1972; Wall et al., 2002). With respect to variation across the visual field the common finding is a small but rather systematic increase of RTs towards the periphery (on average 1.8 ms/deg; Schiefer, Strasburger, et al., 2001). This finding was confirmed in the Toelz Temporal Topography Study which showed a small but steady increase of RTs over the inner $20^{\circ}$ radius (by $1.6 \mathrm{~ms} / \mathrm{deg}$ ), i.e. much shallower than that for the DPR (Poggel et al., 2004a; Poggel et al., submitted). The mean (across the visual field) increase of RTs with increasing age in healthy adults is also steeper than the mean increase in DPR. At the same time, the increase of RTs happens in a more homogenous fashion, i.e. all visual field positions are affected in the same way in contrast to the stronger aging effect in the periphery for DPR (Bellis, 1933; Poggel et al., 2004a; Poggel et al., submitted).

Earlier studies have shown that RTs are elevated in areas of residual vision at the border of blind areas in patients with partial visual field loss (Poggel, 2002; Poggel, Kasten, Mueller-Oehring, Bunzenthal, \& Sabel, 2006; Poggel, Kasten, \& Sabel, 2004). Hence, increased RTs may be an indicator of visual impairment even in areas outside a scotoma, similar to findings from non-conventional mapping studies described above (Bachmann and Fahle, 2000; Castelo-Branco et al., 2006; Castelo-Branco et al., 2009a).

Differences in the topography and variation over the life span between DPR thresholds and RTs suggest that these two temporal parameters of visual information processing reflect partly separate mechanisms (Poggel et al., 2004b; Poggel et al., submitted). We were therefore interested whether DPR thresholds and RTs are elevated in patients with visual field loss. To put these results into perspective we also determined the degree of similarity between the visual field distributions of RT and DPR and compared those topographies to perimetric maps. Deficits in the temporal characteristics of information processing might explain some of the perceptual difficulties in perimetrically intact visual field regions that are experienced by patients with visual field loss. For example, this phenomenon frequently occurs in glaucoma patients with respect to color perception and motion detection (Greenstein et al., 1996; Horn et al., 1999; Shabana et al., 2003; Tyler, 1981). Thus, the presence of functional loss before light detection deficits are detected in perimetry (like in glaucoma) or deficits in higher-order processing like temporal perception in areas clearly outside the perimetrically measured defects are of high diagnostic relevance. More generally, a dissociation between deficits of light perception and of temporal information processing on the behavioral level would contribute to the understanding of how closely those functions and their underlying neural structures are connected. Thus, in addition to the practical clinical value of the study, we expect insight on a basic visual neuroscience level regarding parallel distributed visual processing.

\section{2. $\quad$ Results}

The grand mean DPR threshold (i.e. the minimal perceivable temporal gap between the double pulse targets) over all patients and test positions was $66.8 \mathrm{~ms} \pm 6.6 \mathrm{~ms}$ SEM. DPR thresholds (averaged over the test positions of same eccentricity) increased significantly with eccentricity (Friedman Test $\chi^{2}=19.8$; df $=3 ; \mathrm{p}<0.001$; Table 1, Fig. 2). As in the healthy group, the increase was mostly brought about within the very center. Regression analysis of the raw data yielded a small but significant overall linear trend $\left(R^{2}=0.11\right.$, $\mathrm{p}=0.047$ ) with a slope of $1.03 \mathrm{~ms} / \mathrm{deg}$.

The grand mean of RT over all patients and visual field positions was $466.5 \mathrm{~ms} \pm 31.7 \mathrm{~ms}$ SEM. RT remained almost constant in the inner 10 degrees of the visual field but showed some increase between $10^{\circ}$ and $20^{\circ}$ (Table 1). Overall the increase was $34 \mathrm{~ms}(1.7 \mathrm{~ms} / \mathrm{deg})$, but the differences across eccentricities were not significant due to the high variance (Friedman Test $\chi^{2}=6.7 ; \mathrm{df}=3 ; \mathrm{p}<0.081$ ). Accordingly, regression analysis did not show a significant linear trend $\left(\mathrm{R}^{2}=0.02, \mathrm{p}=0.41\right)$. The same pattern of results, i.e. an increase of DPR thresholds and RT with eccentricity, was found within the intact field and within the defective field when averaged separately (Fig. 1).

\begin{tabular}{|c|c|c|}
\hline $\begin{array}{l}\text { Eccentricity } \\
\text { (deg visual angle) }\end{array}$ & $\begin{array}{l}\text { Mean DPR } \\
\text { ms }( \pm \text { SEM })\end{array}$ & $\begin{array}{l}\text { Mean RT } \\
\text { ms ( } \pm \text { SEM })\end{array}$ \\
\hline 2.5 & $56.8 \pm 7.7$ & $457.0 \pm 33.9$ \\
\hline 5 & $65.8 \pm 7.4$ & $458.0 \pm 33.0$ \\
\hline 10 & $67.3 \pm 7.0$ & $459.3 \pm 34.8$ \\
\hline 20 & $77.3 \pm 5.1$ & $491.6 \pm 32.0$ \\
\hline All peripheral positions & $66.8 \pm 6.6$ & $466.5 \pm 31.7$ \\
\hline
\end{tabular}



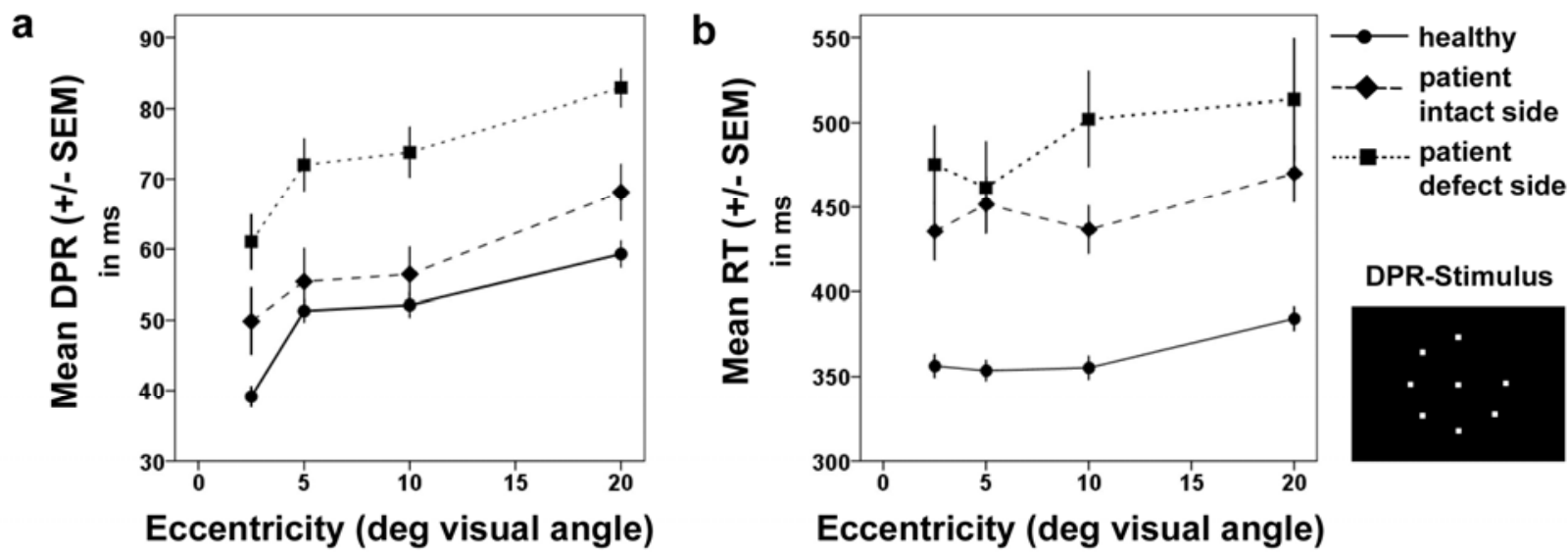

Fig. 1 - DPR and RT over eccentricity. Eccentricity plots of a) mean DPR thresholds, and b) reaction times. Dashed and dotted lines show the intact and defective hemifield of the patients, respectively; solid lines show data for healthy subjects from the Toelz Temporal Topography Study for comparison. Error bars denote SEM. The inset shows an example of a DPR stimulus during the temporal gap of a target in the upper right quadrant.

DPR thresholds and R'Ts were highly correlated across subjects: Global correlations (Table 2, first row) with performance averaged at same eccentricity or globally within the subject prior to correlating, were highest in the center of the field. The topographical correlation (for each position in the visual field correlation of each DPR threshold of each subject with the RT of the same subject at that position, see Methods) was only slightly lower but still substantial (Table 2 , second row). In sum, patients who performed low in one variable (globally, at a certain eccentricity, or at a given position) also performed low in the other, such that the two variables are predictive of each other. To test in how far the common variance of DPR and RT due to the eccentricity in the visual field explains the correlation between these variables, we performed a partial correlation analysis. As expected, the correlation was not reduced, excluding eccentricity as a factor.

Comparisons between the intact and lesioned visual field showed the expected differences: Mean DPR thresholds in the defective hemifield (measured in areas with residual vision on the defective side) were significantly higher than in the intact hemifield (mean DPR threshold intact: $57.5 \mathrm{~ms} \pm$ 3.9 SEM.; defect: $72.0 \mathrm{~ms} \pm 3.6$; Wilcoxon-Test: $Z=5.1, \mathrm{p}<$ 0.001; Figure 2). For RTs, the corresponding difference between the intact and defective field were similar but just missed significance (mean RT intact: $449.4 \mathrm{~ms}$ ( \pm 12.8); defect: $470.9 \mathrm{~ms}( \pm 17.5)$, Wilcoxon-Test: $Z=1.8, \mathrm{p}=0.066$; Figure 2). Note that reaction times were recorded for detected stimuli only (i.e. while areas of residual vision were included, completely blind regions were left out).

Performance depended systematically on the functional status of the particular visual field region (Figure 3), i.e. the lower the light detection performance at a position, the higher the DPR threshold, and the longer the RT. The differences are significant as tested by the Kruskal-Wallis Test (DPR: $\chi^{2}=74.4, \mathrm{df}=5 ; \mathrm{p}<0.001$; RT: $\chi^{2}=58.0, \mathrm{df}=$ 4; $\mathrm{p}<0.001)$.

Not surprisingly, patient DPR thresholds were overall elevated over the norm group performance $(66.8 \mathrm{~ms} \pm 4.7$ $\mathrm{ms}$ versus $49.7 \mathrm{~ms} \pm 1.4 \mathrm{~ms}, \mathrm{p}=0.01$, Figure $2 \mathrm{a})$. In the intact field, however, patient DPR thresholds were close to normal $(57.5 \mathrm{~ms} \pm 7.2 \mathrm{~ms})$, the slight $7.8 \mathrm{~ms}$ difference to healthy subjects not being significant in a Mann-Whitney Test $(\mathrm{p}=0.241)$. RTs, in contrast, were not only elevated overall (RTs patients: $466.5 \pm 31.7 \mathrm{~ms}$; healthy subjects: $370.9 \mathrm{~ms} \pm 7.1 \mathrm{~ms}, \mathrm{p}=0.001$, Figure $2 \mathrm{~b}$ ), but also with respect to the patients' intact visual fields (RTs patients intact areas: $449.4 \mathrm{~ms} \pm 25.2 \mathrm{~ms}$, Wilcoxon $\mathrm{Z}=3.2, \mathrm{p}=0.001)$.

The average increase of DPR thresholds with eccentricity in the visual field was $1.17 \mathrm{~ms} / \mathrm{deg}$. This rate is quite similar to the average increase found for healthy subjects in the Toelz Temporal Topography Study (1.16 ms/deg). Patients' RTs increased at a rate of $1.98 \mathrm{~ms} / \mathrm{deg}$, and again this was similar to the rate found in the healthy sample $(1.60 \mathrm{~ms} / \mathrm{deg})$. Thus, while the absolute level of temporal-processing performance was different between

Table 2 - Correlations between DPR thresholds and RTs. First row: Performance averaged per subject over rings of constant eccentricity (or in last column over all test positions) prior to correlation. Second row: Mean-over-subjects of topographical correlations.

\begin{tabular}{|c|c|c|c|c|c|}
\hline \multirow[t]{2}{*}{ Correlation parameter } & \multicolumn{4}{|c|}{ Eccentricity } & \multirow[t]{2}{*}{ All positions } \\
\hline & $2.5^{\circ}$ & $5^{\circ}$ & $10^{\circ}$ & $20^{\circ}$ & \\
\hline Mean over subjects of Spearman's Rho & $\begin{array}{c}0.98 \\
<0.001\end{array}$ & $\begin{array}{c}0.92 \\
<0.001\end{array}$ & $\begin{array}{c}0.90 \\
<0.001\end{array}$ & $\begin{array}{l}0.67 \\
0.05\end{array}$ & $\begin{array}{c}0.97 \\
<0.001\end{array}$ \\
\hline Topographical correlations (Spearman's Rho) & $\begin{array}{l}0.83 \\
<0.001\end{array}$ & $\begin{array}{l}0.84 \\
<0.001\end{array}$ & $\begin{array}{l}0.78 \\
<0.001\end{array}$ & $\begin{array}{l}0.72 \\
<0.001\end{array}$ & $\begin{array}{l}0.83 \\
<0.001\end{array}$ \\
\hline
\end{tabular}


a
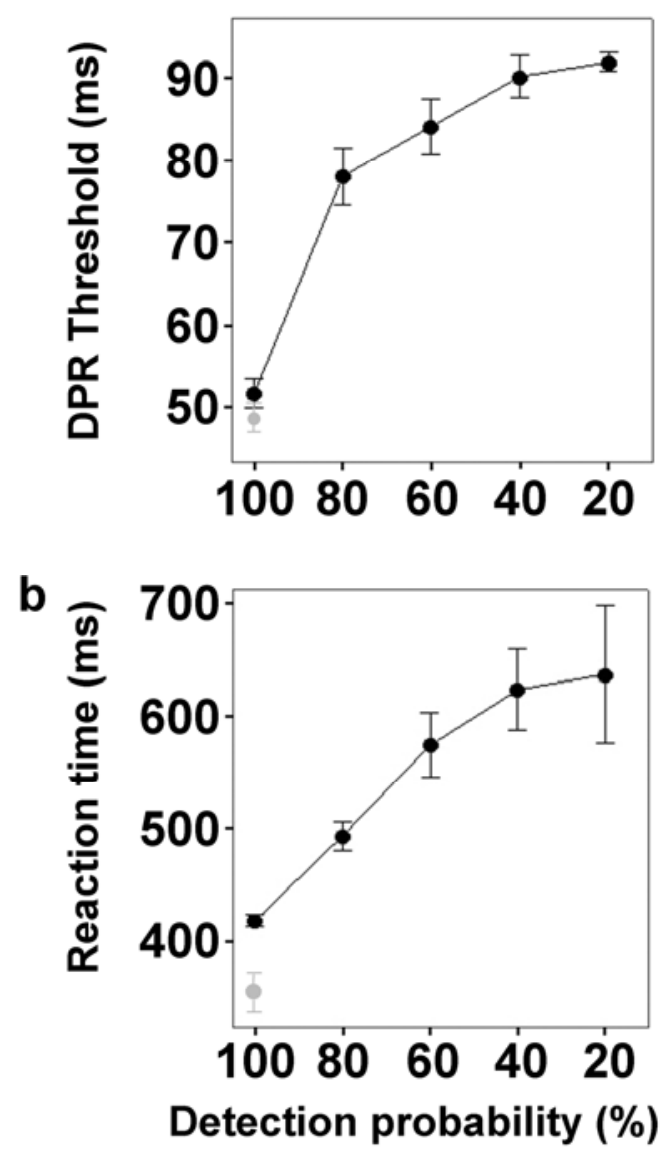

Fig. 2 - DPR and RT over light detection performance (functional status). Performance across visual field areas of decreasing detection probability, i.e. decreasing functional status, as derived from the HRP tests, for a) DPR and b) RT. Grey circles at the $100 \%$ mark show the average performance of healthy subjects from the Toelz Temporal Topography Study for comparison. Error bars denote SEM over subjects.

patients and healthy subjects, the variations across the visual field were comparable between the groups

Since the degree of visual impairment and test performances varied considerably between patients, we also compared individual DPR and RT values to the average performance of each patient's normative age group (Table 3).
While some patients were clearly below normal performance, for both DPR and RT, there were others who in their intact areas showed comparable or even better performance than the age-norm.

Figure 4 shows topographic maps of the three main performance measures for

each of our nine patients. Both topographic similarities and differences between the measures are seen. For instance, Patient 5 with an upper-left quadrantanopia shows almost exactly the same topographical pattern of performance on all variables, i.e. his maps of HRP, threshold perimetry, DPR, and RTs look very similar. In contrast, the maps of Patient 7 for these variables are incongruent (note the difference between HRP/ perimetry and DPR).

\section{Discussion}

In our everyday experience, vision is our most important spatial sense. The significance of the spatial aspect sometimes obliterates the fundamental importance and excellent capacity of the visual system for temporal processing. This is mostly also true for clinical testing of patients with vision loss: conventional light detection perimetry is the gold standard for determining visual performance across the visual field, i.e. performance in the spatial dimension. For patients with post-geniculate lesions, temporal parameters are not part of standard examination procedures. One of the reasons may be that - so far - no specific treatment follows from impairments in the temporal domain. To understand impairments in everyday visual tasks, however, particularly temporal resolution and motion perception, the temporal domain is as important as the spatial aspect of vision (Bachmann and Fahle, 2000; Castelo-Branco et al., 2006; McKendrick, 2005; Myers et al., 2000; Rota-Bartelink, 1999). For example, even without visual field loss, patients with Parkinson's Disease show deficits in motion perception and visual search and were shown to be impaired in their driving abilities (Ergun et al., 2006).

Previous research with healthy subjects has demonstrated that perimetric maps of light- detection thresholds and maps of temporal variables differ considerably (Poggel et al., 2004a; Poggel and Strasburger, 2004). This is in line with previous research showing incongruent topographies for different visual functions in healthy and visually impaired subjects (Bachmann and Fahle, 2000; Castelo-Branco et al.,

Table 3 - Comparison of individual patient's DPR and RT performances with values of the respective age group from the Toelz Temporal Topography Study. Shaded cells indicate significantly lower performance of the patient or his/ her norm group, respectively.

\begin{tabular}{|c|c|c|c|c|c|c|}
\hline $\begin{array}{l}\text { Patient \#, } \\
\text { age group (years) }\end{array}$ & $\begin{array}{l}\text { Mean indiv. DPR } \\
(\mathrm{ms}) \\
\pm \mathrm{SEM}\end{array}$ & $\begin{array}{l}\text { Mean norm. } \\
\text { DPR (ms) } \\
\pm \text { SEM }\end{array}$ & $\mathrm{p}$ & $\begin{array}{l}\text { Mean indiv. RT } \\
(\mathrm{ms}) \\
\pm \text { SEM }\end{array}$ & $\begin{array}{l}\text { Mean norm. } \\
\text { RT (ms) } \\
\pm \text { SEM }\end{array}$ & $\mathrm{p}$ \\
\hline \#1: norm 50 & $64.6 \pm 3.3$ & $48.3 \pm 1.1$ & $<0.001$ & $441.3 \pm 14.1$ & $347.2 \pm 4.4$ & $<0.001$ \\
\hline \#2: norm 30 & $71.8 \pm 2.8$ & $44.9 \pm 1.3$ & $<0.001$ & $526.0 \pm 17.4$ & $339.0 \pm 5.5$ & $<0.001$ \\
\hline \#3: norm 60 & $53.7 \pm 7.0$ & $58.3 \pm 1.2$ & $<0.001$ & $446.2 \pm 16.8$ & $365.3 \pm 4.8$ & $<0.001$ \\
\hline \#4: norm 40 & $88.8 \pm 1.5$ & $49.0 \pm 1.6$ & $<0.001$ & $562.0 \pm 16.5$ & $351.4 \pm 4.8$ & $<0.001$ \\
\hline \#5: norm 40 & $46.9 \pm 2.7$ & $49.0 \pm 1.6$ & 0.186 & $416.0 \pm 11.9$ & $351.4 \pm 4.8$ & $<0.001$ \\
\hline \#6: norm 20 & $22.1 \pm 1.4$ & $41.4 \pm 1.0$ & $<0.001$ & $341.1 \pm 5.8$ & $349.5 \pm 6.7$ & 0.211 \\
\hline \#7: norm 40 & $41.1 \pm 3.0$ & $49.0 \pm 1.6$ & $<0.001$ & $364.5 \pm 8.3$ & $351.4 \pm 4.8$ & 0.008 \\
\hline \#8: norm 20 & $84.3 \pm 2.2$ & $41.4 \pm 1.0$ & $<0.001$ & $525.0 \pm 12.5$ & $349.5 \pm 6.7$ & $<0.001$ \\
\hline \#9: norm 50 & $43.9 \pm 2.2$ & $48.3 \pm 1.1$ & $<0.001$ & $421.3 \pm 13.9$ & $347.2 \pm 4.4$ & $<0.001$ \\
\hline
\end{tabular}




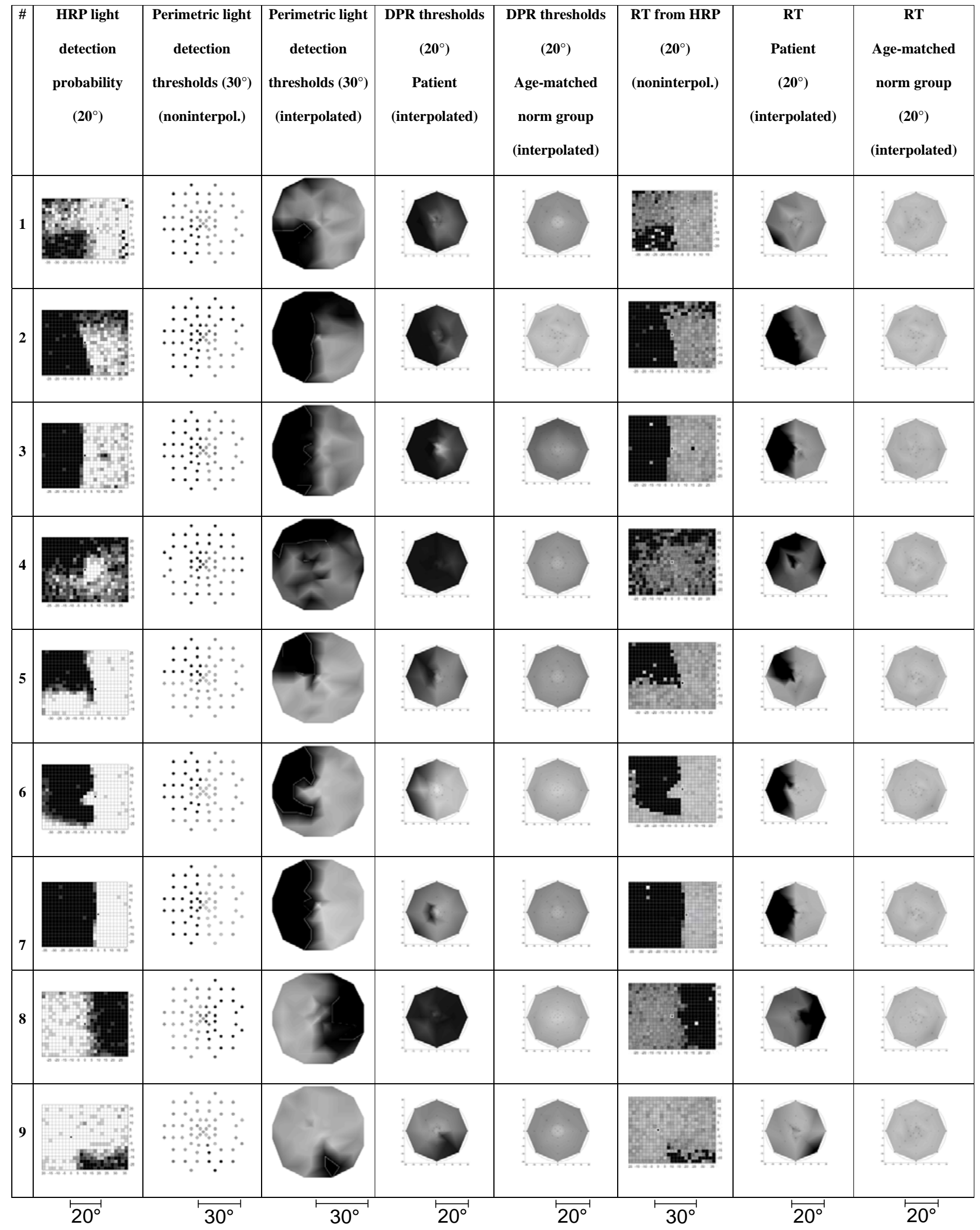

Fig. 3 - Single patients' perimetric, DPR, and RT maps compared to their respective healthy age groups. Individual maps of each patient, and corresponding normative maps of the respective healthy age group from the Toelz Temporal Topography Study: (1) high-resolution perimetry (HRP), (2) threshold perimetry (Oculus, 30 ${ }^{\circ}$ ) noninterpolated and (3) interpolated, (4) double-pulse resolution (DPR) non-interpolated and (5) interpolated, and (6) patient reaction times (RTs, $20^{\circ}$ ) in high resolution and (6) low resolution, compared to (7) age-matched controls. Fields are drawn to size; a scale is shown at the bottom. 
2006; Silva et al., 2008). In addition, we found a high variability between patients with respect to the congruency of visual field maps.

Thus, although light detection and temporal processing appear inseparably connected in the healthy visual system and in our subjective visual experience, their underlying neural mechanisms may be distinct enough to make a dissociation of functional loss possible, e.g. through (partial) damage to networks supporting temporal processing. The functional dissociation therefore points to parallel processes in temporal visual perception, which is interesting because although separate modules and parallel processing have been postulated for various visual functions (e.g., Zeki, 1998) our results point to parallel distributed representation within a conceptual module of temporal processing in the visual system. Each "sub-module" of temporal perception may actually have a closer connection to basic light perception than to other sub-modules of temporal processing.

Selective loss of temporal-processing capacity after brain lesions may at least in part explain patients' complaints, e.g. a reported overwhelming speed of visual impressions, which cannot be traced down by conventional perimetric methods. Such dissociations between temporal functions and light detection performance have been found in patients with retinal or pre-geniculate damage to the visual system (Castelo-Branco et al., 2006; Horn et al., 1999; RotaBartelink, 1999; Wall et al., 2002).

\subsection{Double-pulse resolution and light detection performance}

In our patients with post-geniculate damage to the visual pathway, we found an increase of DPR thresholds with eccentricity. This effect does not stem from motor components of processing speed because DPR measurement involves unspeeded verbal responses only (Poggel et al., 2004a; Poggel and Strasburger, 2004; Poggel et al., 2006b; Poggel et al., submitted). The pattern of a steep increase in the central $5^{\circ}$, and a shallower incline beyond, was the same in patients as in the healthy sample of our earlier study, only with higher overall DPR thresholds in patients. This pattern was even preserved in the defective hemifield of the patient sample, again with thresholds being higher than in the intact field. In a study on double-pulse resolution in healthy subjects, we had argued that the increase of DPR thresholds towards the periphery was in part due to the test stimuli not being scaled in size with the cortical magnification factor (Poggel et al., 2006b, Appendix; based on Tyler \& Hamer, 1990). In the same study, we had shown that the larger attention focus required to simultaneously monitor all test positions with increasing stimulus eccentricity also contributed to the increase of thresholds towards the periphery. The striking similarity of the pattern of increase in normal subjects, on the intact side of the visual field in patients, and on the patients' defective side, suggests that DPR thresholds may be influenced by the same two factors in patients, i.e. cortical magnification and the size of the attention focus. Interestingly, the presence of a visual field defect does not distort the influence of those factors. Instead, the lesion appears to operate like a gain reduction, decreasing the overall level of performance without changing the pattern of DPR increase over eccentricity.

This view was supported by the significant differences between DPR thresholds in regions of differing perimetric detection probability (i.e. levels of impairment, see Figure 3).
Thus, there seems to be a quantitative connection between simple detection of light stimuli (as required in perimetric testing), and double-pulse resolution. Internal noise in the lesioned visual system that underlies impeded light detection in areas of residual vision might thus also affect the detection of a gap between two light pulses. In Poggel, Treutwein et al. (2006, Fig. 6) we note a hypothetical mechanism based on the signal-to-noise ratio that is compatible with such an interpretation (cf. Pelli \& Farell 1999).

Most interestingly, however, DPR may also be impaired although perimetrically the respective visual field region seems intact or almost so. Within the general correlation of DPR and perimetry (Fig. 3), there were large interindividual differences when the patient data were individually compared to the age-matched healthy subject group (Fig. 4). While some patients showed lower-than-normal performance in both perimetrically intact and impaired regions, others showed deficits of temporal resolution only around the visual field border but performed even better than the age-matched controls in the intact field. These findings emphasize the need for additional testing of temporal visual functions in addition to conventional perimetry to determine the presence and location of deficits in temporal resolution which can be partly or fully dissociated from light detection performance.

\subsection{Reaction times and light detection performance}

RTs in the patient sample increased slightly and monotonously towards the periphery of the visual field. Such an increase of RTs with eccentricity has been found for normally-sighted subjects (Poggel et al., 2004a; Poggel and Strasburger, 2004; Schiefer et al., 2001; Poggel et al., submitted). The increase rate for RTs was lower than that observed for the DPR thresholds and, particularly in the central $5^{\circ}$, less steep than for DPR, but very reliable. This pattern was found both in the intact and defective visual hemifields of our patients. Thus, like in DPR, the variation of performance with eccentricity seems to be governed by the same, sensory factors in the unaffected and the lesioned hemisphere. Whether the slowing of RT in some patients' perimetrically intact areas stems from sensory or motor factors or (a rather improbable) interaction of the two cannot be decided based on our data. The observation that DPR thresholds are also increased in patients would suggest that there is a sensory component in the patients' R'T slowing.

Similar to DPR, RTs depended very systematically on perimetric defect depth of a specific visual field position. Longer RTs with increasing impairment of the visual field could reflect a weakened strength of the neural signal, leading to a more difficult decision regarding the presence of a light stimulus. In the partially defective regions at the visual field border RTs were markedly increased, but the interindividual variability was so high that the overall difference between the intact and defective hemifields just missed significance. Compared to their age groups in a normally sighted sample, all but one patient showed slower RTs which may be due to general slowing in response to the brain lesion or specific residual motor deficits.

\subsection{Relationship of DPR and RTs as indicators of temporal information processing}

The overall correlations between DPR and RTs were rather high and hide the differences in the topographical 
distributions. Based on how the measures are obtained, this simply reflects the large variance between subjects, i.e. subjects with good performance in one measure also show good performance in the other (e.g. based on common influence of cognitive factors like attention, see also Carrasco et al., 2006; Castelo-Branco et al., 2009a). Still, even with high overall correlation values, the form of DPR and RT maps can be very different within a patient which is reflected in the comparatively low topographical correlations (covariation of RTs and DPR thresholds at every single position in the visual field). In normally sighted subjects, the between-subject intercorrelation of DPR and RTs is significant but much lower (Rho $=0.314$, see Poggel, Calmanti et al., 2004) than the correlation observed in the patient sample. We ascribe this to the lower intersubject-variance of the measures in the healthy group. Even with significant correlations between DPR and RT there is still room for non-commonly explained variance and thus dissociations across measures that may be of practical relevance.

The relatively high overall correlation between DPR thresholds and RT cannot be explained by the visual field position: on the one hand, the topographical correlation was low, on the other hand, the correlation between DPR thresholds and RT was unchanged by partialling out eccentricity as a factor.

\section{Temporal processing in the lesioned visual system}

There are several potential explanations for deficits of temporal processing in patients with lesions of the visual system. Temporal processing performance as measured by DPR and RT strongly depends on attention in normally sighted subjects (Poggel et al., 2006) so that the deficits observed in patients might be caused by cognitive deficits which are common after brain lesions. However, we do not believe that the results can be explained by attention problems of our patients. The form of the eccentricity distribution of RT and DPR is similar between patients and healthy subjects and also similar on the intact and lesioned side of the same patient. The difference is quantitative rather than qualitative, i.e. RTs and DPR thresholds are increased by an additive factor so that the map distributions as such are not changed by the lesion.

We believe that the problem is based on a decreased signal-to-noise ratio in the lesioned visual system. For DPR thresholds, we hypothesized, based on physiological findings on how light pulses can be coded in afferent fibers (Fain \& Cornwall, 1993), that a central read-out mechanism detects the temporal gap in the light stimulus (Poggel et al., 2006b). This mechanism is assumed to fail when there is too much noise in the system. This increase of noise is known to be the present in areas of residual vision around the visual field defect where perceptual thresholds are higher and further spontaneous visual activations (e.g. illusions and pseudohallucinations) frequently occur.

For RT, the problem might have different causes, depending on the lesion site. After brain lesions, patients are often generally slowed, including prolonged motor RT, but also delayed responses to sensory stimulation. On the one hand, this may be due to reduced transmission speed of neurons in those brain areas that represent areas of residual vision, e.g. after partial damage to the optic nerve. On the other hand, there may be a top-down explanation for the increased RT: the interpretation of incoming visual stimulation becomes more difficult due to the lower signalto-noise ratio, i.e. the decision whether or not there was a visual stimulus takes longer.

\section{Conclusions}

In summary, differences within and between patients with respect to temporal-information processing may be an indicator for a higher-order visual impairment that cannot be measured by conventional perimetry alone. Many patients with relatively large perimetrically intact regions describe problems with scanning the visual environment quickly, or report their impression of a "sluggishness" of perception. Our finding may hence indicate that - even if parts of the visual field had remained perimetrically intact or have recovered after visual system lesions (Kasten et al., 1998; Kasten et al., 2006) - there may be still other levels of visual processing affected by the lesion that could induce considerable difficulties handling the visual environment.

We suggest that tests of temporal-information processing should be included more routinely, i.e. also for patients with post-geniculate damage, in visual clinical diagnostics. In addition to clarifying the connection between basic visual processing and mechanisms of temporal-information processing in the healthy and lesioned visual system, this may be a useful basis for visual rehabilitation. We predict that light detection training (designed to increase intact visual field size in patients with vision loss) should also improve parameters of temporal processing. And even stronger effect should be expected from specific training of temporal performance.

\section{Experimental Procedure:}

\section{$5.1 \quad$ Sample}

Nine patients (three female) with visual field loss were recruited for the study. The mean age in the sample was 42 years ( \pm 4.5 years; range $22-62$ years). One patient had a pregeniculate lesion of the optic nerve due to tumor surgery (close to the optic chiasma); the others had post-geniculate visual system lesions. Six of the latter suffered stroke of the posterior or middle artery, one had had a brain trauma (widespread lesions with an emphasis on the left hemisphere), and one had undergone aneurysm surgery (left posterior artery). In the eight patients with post-geniculate lesions, the right hemisphere was affected in six and the left hemisphere in two patients. Exclusion criteria for the study were dementia, spatial hemi-neglect, severe attentional deficits (especially vigilance), depression and other psychiatric disorders, as well as visual impairment resulting from ophthalmic diseases. Information on dementia, psychiatric disorders and ophthalmic diseases were extracted from the patient files. Standard neuro-ophthalmological and neuropsychological tests were used for ruling out the presence of the other exclusion criteria: We screened visual functions like acuity, contrast perception, $3 \mathrm{D}$ perception, and performed a conventional perimetric test. We also performed an Alertness test (TAP, Test Battery for Attentional Performance, Zimmermann \& Fimm, a computer-based test that is widespread in German neuropsychological settings). 
In addition, patients were screened for object recognition deficits (VOSP), attentional interference (Stroop), and visual exploration (German version of the trail making test). We used the BIT (Behavioural Inattention Test, Wilson, Cockburn \& Halligan) to test for neglect.

Testing for pre-screening and data acquisition were performed in five to six relatively short sessions to reduce the strain of testing for the patients and potential fatigue. Generally, the experimenter was very observant of potential fatigue of the patients and suggested breaks even when the patients felt that they could go on

All subjects gave their informed consent for participating in the study. The experimental design had been approved by the local ethics committee and was in accordance with the guidelines of the Declaration of Helsinki.

\subsection{Double-pulse resolution}

The apparatus and psychophysical technique for the measurement of double-pulse resolution had originally been developed by Treutwein (1989; Treutwein and Rentschler, 1992; Treutwein, 1995; Treutwein, 1997). The general settings and procedure for the test have been described elsewhere (Poggel et al., 2004a; Poggel and Strasburger, 2004; Poggel et al., 2006b). In brief, the patient was sitting in a darkened room (illuminance $=1.5 \mathrm{~lx}$ ) at $30 \mathrm{~cm}$ viewing

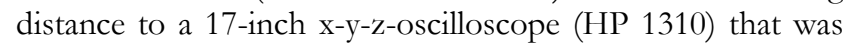
controlled by a so-called point plot buffer (Finley, 1985) which in turn received its input from an IBM-compatible PC. With this unique setup, stimulus presentation and adaptation of the gap duration can be controlled with microsecond accuracy since it circumvents raster-scan or TFT/ LCD technology (see Bach et al., 1997; Otto et al., 2006; Treutwein, 1989).

The subject's head was positioned on a chin rest with the eyes located opposite the center of the screen. A cross-hair was displayed before each trial. Nine rectangular white light stimuli (luminance: $215 \mathrm{~cd} / \mathrm{m}^{2}$, size: $1.15^{\circ}$ ) were then presented simultaneously on dark background, one in the center, and the others on a circle around it at the intersections with the main horizontal, vertical and $45^{\circ}$ meridians (see inset in Fig. 2). Eight of the nine stimuli within a trial served as distracters and were presented continuously, while the target was interrupted by a temporal gap. For gap durations above the threshold, the target could be discriminated from the distracters by a perceived short flickering of that stimulus. The subject verbally indicated the target position, and the response was entered by the experimenter on the computer keyboard. Subjects were instructed to keep their eyes fixated at the central position of the cross hair displayed between trials. Fixation was controlled in all trials by the experimenter observing the subject's eye position via a conventional (ca. $15 \mathrm{~cm}$ diameter) mirror positioned next to the computer screen in the patient's blind field to avoid distraction. The experimenter (DAP) observed the eyes of the patient (clearly visible thanks to the luminance of the computer monitor and the dim surrounding light) throughout the tests. Both large eye movements towards an eccentric stimulus (note that presentation time was below saccade onset time) and small "scanning" saccades some patients made in the beginning could be clearly identified. Feedback was given in these cases, and all patients managed to keep their eyes still. Eccentric fixation could be identified by instable fixation when the patient tried to fixate in the empty visual field somewhere beyond the fixation point. In addition, fixation was controlled with an eye-tracking device (IViewX, Sensomotoric Instruments, Teltow, Germany) yielding a spatial resolution of below one degree visual angle. For one patient the device had not been available yet, and for the remainder of the sample $50-80 \%$ of measurements were accompanied by automatic eye tracking on top of control of eye position via the mirror. Subjects were asked to indicate the target position on each trial, even when guessing. The new trial was started when the subject was ready, and fixation stable at the center of the screen.

The YAAP maximum-likelihood thresholding algorithm controlled the gap duration between the two light pulses of the target. The starting point was set to $80 \mathrm{~ms}$ which was well above threshold for intact positions in the visual field. DPR thresholds were determined independently of each other in an interleaved fashion; target positions varied randomly from trial to trial. The first light pulse of the target patch had 80 ms duration, the second (i.e. after the gap) $280 \mathrm{~ms}$ (see Treutwein, 1989; Treutwein and Rentschler, 1992, for details on stimulus parameters). The distracters were presented simultaneously with the target so that their entire duration matched that of the target stimulus including the gap. Targets and non-targets appeared equal in brightness since they were above the summing duration in Bloch's law (Treutwein, 1989; Treutwein and Rentschler, 1992).

A test block was ended when all nine thresholds were determined to a pre-specified confidence interval containing the threshold at $85 \%$ probability; this took approximately 140 - 280 trials (between ten and twenty minutes test duration). Eight blocks of trials were performed per subject. Within a block, the eccentricity of the peripheral stimuli, i.e. the ring radius, was constant. Four blocks with ascending ring radius, $2.5^{\circ}, 5^{\circ}, 10^{\circ}$, and $20^{\circ}$, respectively, were conducted, followed by another four blocks in reverse order of eccentricities such that each eccentricity block occurred twice. Prior to the first trial of each block, the positions of the stimuli in that block were shown to the subjects. In most subjects, the two series of four blocks were done in separate sessions, within an interval of a few days.

Test speed and duration were under the control of the subjects by answering in a self-paced manner. Participants were also free to take breaks whenever they wished. In an initial set of practice trials which included feedback from the experimenter, the subject learned to recognize the flicker targets. In the experiment proper, no feedback was given so as to not bias the threshold measurements.

Raw threshold values (of individual subjects or averaged over the complete sample, see Results) were entered into statistical software for data analysis (Microsoft Excel and SPSS Version 12, Chicago, IL). The 3-D plots were prepared with a Matlab script originally programmed by K. Lutz (see Gothe et al., 2000). The code was modified for the present purpose to get a graphic display of the double-pulse resolution map by means of linear interpolation between average values at all target positions (Matlab Version 5.3, The Mathworks, Natick). 


\section{a}

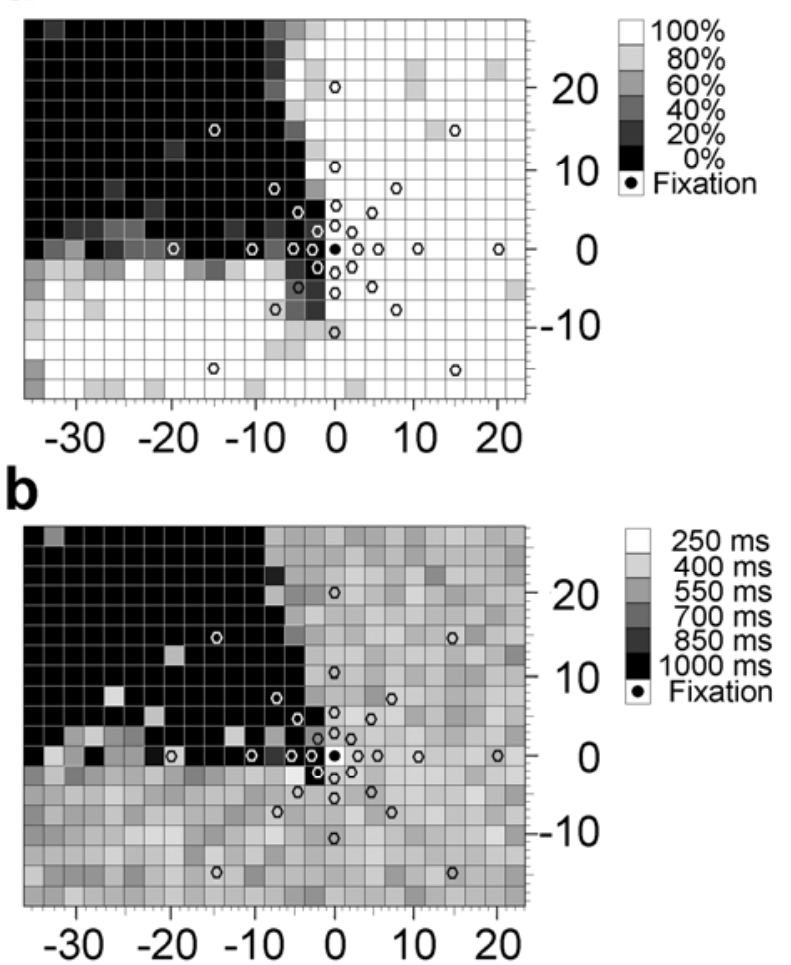

Fig. 4 - Location of DPR stimulus positions relative to the visual field maps. a) A narrow area of residual vision is seen around the patient's blind field in the left-upper quadrant (five superimposed measurements of HRP campimetric light detection test). b) RT map for the same patient, determined by high-resolution perimetry (HRP). Open hexagonal markers in (a) and (b) show the DPR measurement positions. For data analysis, only the corresponding data from those positions were used.

\subsection{Light detection and reaction time maps}

Visual field maps were acquired by conventional static perimetry (Octopus 101 Perimeter, Interzeag/ Haag Streit, Koeniz-Berne, Switzerland). In addition, the detailed light detection maps and reaction time (RT) maps that were used for the comparison with DPR thresholds were obtained using a high-resolution computer-based campimetric test (HRP, Nova Vision Inc., Boca Raton, FL). The examination was performed under the same standardized conditions as described above for DPR testing. A personal computer with a 17" screen was used for stimulus presentation (viewing distance: $30 \mathrm{~cm}$, horizontal size: $\pm 29^{\circ}$, vertical size: $\pm 23^{\circ}$ ). Viewing was binocular in all patients except in the subject with optic nerve lesion who was tested on his left eye only. Circular white light stimuli (luminance $=96 \mathrm{~cd} / \mathrm{m}^{2}$, size $=$ $0.76^{\circ}$ visual angle, duration $=150 \mathrm{~ms}$ ) were presented in random sequence at 474 positions on the screen $(26 \times 19$ positions, $\pm 29^{\circ}$ horizontally and $\pm 23^{\circ}$ vertically), with a background luminance of $26 \mathrm{~cd} / \mathrm{m}^{2}$.

The fixation mark was positioned on the screen in such a way that about half of the stimuli lay in the blind field. The subject pressed the space bar on the computer keyboard whenever a stimulus was detected. Feedback of correct responses and false alarms, respectively, was provided by a high vs. low tone following the response. Fixation was controlled by requiring the subject to detect a change of the fixation point's color from equiluminant green to yellow (Kasten et al., 1997). Additionally, the eye position throughout the test was always monitored by the experimenter via a mirror and in most cases also recorded with an eye-tracker (see above).

Detection and misses were both registered by the test and used for further analysis. The average of false positive reactions was $0.01 \%$ with one patient reaching up to $1 \%$ in two tests. In most cases these false positive responses were delayed reactions to real light stimuli that occurred right after the $1000 \mathrm{~ms}$ cut-off for the reaction. This occurred always in areas of residual vision where RTs were longer due to the patient's uncertainty whether or not a light stimulus had been presented. False negatives cannot be reliably discriminated from vision loss or lapses of attention and were therefore not included in the analysis. For detected stimuli, the RT was recorded (see Results section; for a more detailed description of the method see (Poggel, 2002; Poggel et al., 2004b; Poggel et al., 2006a).

5.3.1 Stimulus detection performance/ mapping of partially lesioned areas

Each patient performed high-resolution perimetry five times, usually over a period of four to six weeks. Results from five tests were superimposed, and graded response rates were obtained at each location. In particular, areas of residual vision that are typically found at the border of the blind field were mapped as those regions with detection rates between 20\% and 80\% (see Poggel, 2002; Poggel et al., 2004b; Poggel et al., 2006a, and Fig. 1a). Subregions within the latter having one of the four intermediate detection rates of $20 \%, 40 \%$, $60 \%$, or $80 \%$, respectively, were defined separately for later analysis to reflect the severity of lesion or the degree of visual impairment. These same subregions were also used for comparison of DPR thresholds between regions with varying degree of lesion.

\subsubsection{Reaction times}

RTs were recorded for the stimuli that were detected by the subject. Average RTs over five high-resolution perimetry tests were plotted in reaction time maps (Fig. 1b, Fig. 4). The graphic RT-maps were prepared by the Matlab scripts mentioned above. Additionally, RTs were averaged separately for each of the subregions of $20 \%$ to $80 \%$ detection probability, i.e. averaged depending on functional status (see Results section; for a more detailed description of the method see Poggel, 2002; Poggel et al., 2004b; Poggel et al., 2006a).

Note that any variation of RTs across the visual field reflects the sensory component since motor requirements are constant (Schiefer et al., 2001; Teichner and Krebs, 1972; the motor component contributes to the absolute RT level only).

\section{Data analysis}

Raw data from DPR, HRP, and perimetric tests were imported into Excel spreadsheets for preprocessing. DPR values from the first and second test at the same positions were averaged. The latter averaged thresholds were subsequently read into Matlab Software and plotted as described above.

For a global comparison between subjects, the overall average over all visual field positions was determined per 
subject, as well as test performance for the defective and intact hemifield separately. Note that there were usually some intact or partially intact positions remaining even in the defective hemifield so that performance values could be calculated in the latter. To examine the dependency on eccentricity, both within DPR and for comparison with RTs, the averages over all test positions for a specific ring (i.e. a specific eccentricity) were determined. For a topographical comparison between DPR and RT within subjects, for each patient the correlations between the two variables at the corresponding locations were determined

$$
\rho=1-\frac{6 \sum_{i}^{\text {positions }} d_{i}^{2}}{n\left(n^{2}-1\right)}
$$

(where $d_{i}$ are the differences in rank between the two variables at position i), and these correlations were then averaged over subjects. We denote this as mean topographical correlation. To match the less densely sampled DPR positions to those in HRP, only the RT values at corresponding positions were selected from the spreadsheet.

Patient DPR and HRP data were further compared to the average data of healthy controls, i.e. to data from the respective age decade of the patient, based on a large normative study on aging of the visual system (Poggel and Strasburger, 2004; Poggel et al., 2006b).

Nonparametric tests were used to compare average values (Friedman Test, Kruskal-Wallis Test, Mann-Whitney U-Test, Wilcoxon Test). Nonparametric correlations were used to determine the co-variation between variables (Spearman's Rho). Due to the small sample size and since not all test parameters were normally distributed, we preferred nonparametric statistics for significance testing. In addition, linear regression analysis was performed to identify trends in the data, and partial correlations for identifying the common variance of DPR and RT explained by eccentricity. All statistical testing was done with SPSS (Version 12.0, Chicago, IL). The alpha level was set to 0.05 , two-tailed.

\section{Acknowledgements}

The authors thank Dr. Kai Lutz and Dr. Jan Churan for their help with programming scripts for the graphical displays, and Dipl.-Psych. Claudia Calmanti, Andrea Geyer, and Luise Schulze for their help with data acquisition. Sebastian Rummel's technical support was essential for conducting the study. This work was supported by a grant of the Deutsche Forschungsgemeinschaft (Str 354/3-1) to Hans Strasburger.

\section{REFERENCES}

Bach, M., Meigen, T., Strasburger, H., 1997. Raster-scan cathode-ray tubes for vision research - limits of resolution in space, time and intensity, and some solutions. Spat Vis. 10, 403-414.

Bachmann, G., Fahle, M., 2000. Component perimetry: a fast method to detect visual field defects caused by brain lesions. Invest Ophthalmol Vis Sci. 41, 2870-86.

Becker, S.T., Vonthein, R., Volpe, N.J., Schiefer, U., 2005. Factors influencing reaction time during automated kinetic perimetry on the Tubingen computer campimeter. Invest Ophthalmol Vis Sci. 46, 2633-8.
Bellis, C.J., 1933. Reaction time and chronological age. Proceedings of the Society for Experimental Biology and Medicine. 30, 801803.

Callaway, E.M., 2005. Structure and function of parallel pathways in the primate early visual system. J Physiol. 566, 13-9.

Carrasco, M., Giordano, A.M., McElree, B., 2006. Attention speeds processing across eccentricity: feature and conjunction searches. Vision Res. 46, 2028-40.

Castelo-Branco, M., Mendes, M., Silva, M.F., Januario, C., Machado, E., Pinto, A., Figueiredo, P., Freire, A., 2006. Specific retinotopically based magnocellular impairment in a patient with medial visual dorsal stream damage. Neuropsychologia. 44, 23853.

Castelo-Branco, M., Kozak, L.R., Formisano, E., Teixeira, J., Xavier, J., Goebel, R., 2009a. Type of featural attention differentially modulates hMT + responses to illusory motion aftereffects. J Neurophysiol. 102, 3016-25.

Castelo-Branco, M., Mendes, M., Silva, F., Massano, J., Januario, G., Januario, C., Freire, A., 2009b. Motion integration deficits are independent of magnocellular impairment in Parkinson's disease. Neuropsychologia. 47, 314-20.

DeYoe, E.A., Van Essen, D.C., 1988. Concurrent processing streams in monkey visual cortex. Trends Neurosci. 11, 219-26.

Drasdo, N., 1991. Neural substrates and threshold gradients of peripheral vision. In: Limits of vision., J.J. Kulikowski, V. Walsh, I.J. Murray, eds. Macmillan Press, London, pp. 250-264.

Ergun, Y., Rizzo, M., Anderson, S.W., Sparks, J.D., Rodnitzky, R.L., Dawson, J.D., 2006. Impaired visual search in drivers with Parkinson's disease. Ann Neurol, 60 (4), 407-413.

Fain, G.L., Cornwall, M.C., 1993. Light and dark adaptation in vertebrate photoreceptors. In: Contrast Sensitivity: From Receptors to Clinic. R. Shapley, D.-K. Lam, eds. MIT Press, Boston, MA. pp. 3-32.

Finley, G., 1985. A high-speed point plotter for vision research. Vision Res. 25, 1993-1997.

Goodale, M.A., Kroliczak, G., Westwood, D.A., 2005. Dual routes to action: contributions of the dorsal and ventral streams to adaptive behavior. Prog Brain Res. 149, 269-83.

Gothe, J., Strasburger, H., Lutz, K., Kasten, E., Sabel, B.A., 2000. Recognition of low-contrast characters by subjects with cerebral visual-field defects. Perception. 29 Suppl., p.45.

Greenstein, V.C., Halevy, D., Zaidi, Q., Koenig, K.L., Ritch, R.H., 1996. Chromatic and luminance systems deficits in glaucoma. Vision Res. 36(4), 621-629.

Harrington, D.O., 1981. The Visual Fields: A Textbook and Atlas of Clinical Perimetry., CV Mosby, St Louis.

Horn, F.K., Velten, I.M., Junemann, A., Korth, M., 1999. The fullfield flicker test in glaucomas: influence of intraocular pressure and pattern of visual field losses. Graefes Arch Clin Exp Ophthalmol. 237, 621-8.

Horton, J.C., Hoyt, W.F., 1991. The representation of the visual field in human striate cortex. A revision of the classic Holmes map. Arch Ophthalmol. 109, 816-24.

Ivry, R.B., Spencer, R.M., 2004. The neural representation of time. Curr Opin Neurobiol. 14, 225-32.

Kasten, E., Strasburger, H., Sabel, B.A., 1997. Programs for diagnosis and therapy of visual deficits in vision rehabilitation. Spat Vis. 10, 499-503.

Kasten, E., Wüst, S., Behrens - Baumann, W., Sabel, B.A., 1998. Computer-based training for the treatment of partial blindness. Nat Med. 4, 1083-1087.

Kasten, E., Bunzenthal, U., Sabel, B.A., 2006. Visual field recovery after vision restoration therapy (VRT) is independent of eye movements: an eye tracker study. Behav Brain Res. 175, 18-26.

Luce, R.D., 1986. Response times. Oxford University Press, Oxford.

Mauk, M.D., Buonomano, D.V., 2004. The neural basis of temporal processing. Annu Rev Neurosci. 27, 307-40.

McKendrick, A.M., 2005. Recent developments in perimetry: test stimuli and procedures. Clin Exp Optom. 88, 73-80. 
Mendes, M., Silva, F., Simoes, L., Jorge, M., Saraiva, J., CasteloBranco, M., 2005. Visual magnocellular and structure from motion perceptual deficits in a neurodevelopmental model of dorsal stream function. Brain Res Cogn Brain Res. 25, 788-98.

Mishkin, M., Ungerleider, L.G., 1982. Contribution of striate inputs to the visuospatial functions of parieto-preoccipital cortex in monkeys. Behav Brain Res. 6, 57-77.

Myers, R.S., Ball, K.K., Kalina, T.D., Roth, D.L., Goode, K.T., 2000. Relation of useful field of view and other screening tests to on-road driving performance. Percept Mot Skills. 91, 279-90.

Otto, E., 1987. Multifaktorielle Abhängigkeit der kritischen Flimmerverschmelzungsfrequenz

Wirkungszusammenhang physikalischer und physiologischer Einflußgrößen. Z Psychol. 195, 261-281.

Otto, T.U., Ogmen, H., Herzog, M.H., 2006. The flight path of the phoenix--the visible trace of invisible elements in human vision. J Vis. 6, 1079-1086.

Poggel, D.A., 2002. Effects of visuo-spatial attention on the restitution of visual field defects in patients with cerebral lesions. Shaker Verlag, Aachen.

Poggel, D.A., Calmanti, C., Treutwein, B., Strasburger, H., 2004a. The Toelz Temporal Topography Study: Mapping the visual field across the life span. J Vis. 4, 76a.

Poggel, D.A., Kasten, E., Sabel, B.A., 2004b. Attentional cueing improves vision restoration therapy in patients with visual field defects. Neurology. 63, 2069-76.

Poggel, D.A., Strasburger, H., 2004. Visual perception in space and time - mapping the visual field of temporal resolution. Acta Neurobiol Exp. 64, 427-437.

Poggel, D.A., Kasten, E., Mueller-Oehring, E.M., Bunzenthal, U., Sabel, B.A., 2006a. Improving residual vision by attentional cueing in patients with brain lesions. Brain Res. 1097, 142-148.

Poggel, D.A., Treutwein, B., Calmanti, C., Strasburger, H., $2006 \mathrm{~b}$. Increasing the temporal $\mathrm{g}(\mathrm{r})$ ain: double-pulse resolution is affected by the size of the attention focus. Vision Res. 46, 29983008.

Rashbass, C., 1970. The visibility of transient changes of luminance. J Physiol. 210, 165-186.

Rota-Bartelink, A., 1999. The diagnostic value of automated flicker threshold perimetry. Curr Opin Ophthalmol. 10, 135-9.

Schiefer, U., Strasburger, H., Becker, S.T., Vonthein, R., Schiller, J., Dietrich, T.J., Hart, W., 2001. Reaction time in automated kinetic perimetry: effects of stimulus luminance, eccentricity, and movement direction. Vision Res. 41, 2157-64.

Shabana, N., Cornilleau Pérès, V., Carkeet, A., Chew, P.T., 2003. Motion perception in glaucoma patients: a review. Surv Ophthalmol. 48(1), 92-106.

Silva, M.F., Maia-Lopes, S., Mateus, C., Guerreiro, M., Sampaio, J., Faria, P., Castelo-Branco, M., 2008. Retinal and cortical patterns of spatial anisotropy in contrast sensitivity tasks. Vision Res. 48, $127-35$.

Strasburger, H., Rentschler, I., 1996. Contrast-dependent dissociation of visual recognition and detection fields. Eur J Neurosci. 8, 1787-91.

Teichner, W.H., Krebs, M.J., 1972. Laws of the simple visual reaction time. Psychol Rev. 79, 344-58.

Treutwein, B., 1989. Zeitliche Aspekte der visuellen Informationsverarbeitung. Kyrill \& Method Verlag, München.

Treutwein, B., Rentschler, I., 1992. Double pulse resolution in the visual field: The influence of temporal stimulus characteristics. Clin Vis Sci. 7, 421-434.

Treutwein, B., 1995. Minireview: Adaptive psychophysical procedures. Vision Res. 17, 2503-2522.

Treutwein, B., 1997. YAAP: Yet another adaptive procedure. Spatial Vision. 11, 129-134.

Tyler, C.W., 1981. Specific deficits of flicker sensitivity in glaucoma and ocular hypertension. Invest Ophthalmol Vis Sci. 20, 204-12.

Tyler, C.W., 1985. Analysis of visual modulation sensitivity: II. Peripheral retina and the role of photoreceptor dimensions. J Opt Soc Am. 2, 393-398.
Tyler, C.W., 1987. Analysis of visual modulation sensitivity: III. Meridional variations in peripheral flicker sensitivity. J Opt Soc Am. 4, 1612-1619.

Tyler, C.W., Hamer, R.D., 1990. Analysis of visual modulation sensitivity. IV. Validity of the Ferry-Porter law. J Opt Soc Am. 7, 743-758.

Tyler, C.W., Hamer, R.D., 1993. Eccentricity and the Ferry-Porter law. J Opt Soc Am. 10, 2084-2087.

Wall, M., Kutzko, K.E., Chauhan, B.C., 2002. The relationship of visual threshold and reaction time to visual field eccentricity with conventional automated perimetry. Vision Res. 42, 781-7.

Watson, A.B., 1986. Temporal Sensitivity. In: Handbook of Perception and Human Performance. K. Boff, L. Kaufman, J. Thomas, eds. Wiley, New York.

Wittmann, M., 1999. Time perception and temporal processing levels of the brain. Chronobiol Int. 16, 17-32.

Wittmann, M., 2009. The inner experience of time. Philos Trans R Soc Lond B Biol Sci. 364, 1955-67.

Zeki, S., 1998. Parallel processing, asynchronous perception, and a distributed system of consciousness in vision. Neuroscientist. 4 (5), 365-372. 